\title{
Nephrin strands contribute to a porous slit diaphragm scaffold as revealed by electron tomography
}

\author{
Jorma Wartiovaara, ${ }^{1}$ Lars-Göran Öfverstedt,, ${ }^{2}$ Jamshid Khoshnoodi, ${ }^{3}$ Jingjing Zhang, ${ }^{3}$ \\ Eetu Mäkelä, ${ }^{1}$ Sara Sandin, ${ }^{2}$ Vesa Ruotsalainen, ${ }^{4}$ R. Holland Cheng, ${ }^{5,6}$ Hannu Jalanko, ${ }^{7}$ \\ Ulf Skoglund, ${ }^{2}$ and Karl Tryggvason ${ }^{3}$

\begin{abstract}
${ }^{1}$ Institute of Biotechnology, University of Helsinki, Helsinki, Finland. ${ }^{2}$ Department of Cell and Molecular Biology and ${ }^{3}$ Department of Medical Biochemistry and Biophysics, Karolinska Institutet, Stockholm, Sweden. ${ }^{4}$ Biocenter and Department of Biochemistry, University of Oulu, Oulu, Finland. ${ }^{5}$ Department of Biosciences, Karolinska Intstitutet Novum, Huddinge, Sweden. ${ }^{6}$ Department of Molecular and Cellular Biology, UCD, Davis, California, USA. ${ }^{7}$ Hospital for Children and Adolescents, University of Helsinki, Helsinki, Finland.
\end{abstract}

\begin{abstract}
Nephrin is a key functional component of the slit diaphragm, the structurally unresolved molecular filter in renal glomerular capillaries. Abnormal nephrin or its absence results in severe proteinuria and loss of the slit diaphragm. The diaphragm is a thin extracellular membrane spanning the approximately 40 -nm-wide filtration slit between podocyte foot processes covering the capillary surface. Using electron tomography, we show that the slit diaphragm comprises a network of winding molecular strands with pores the same size as or smaller than albumin molecules, as demonstrated in humans, rats, and mice. In the network, which is occasionally stratified, immunogold-nephrin antibodies labeled individually detectable globular cross strands, about $35 \mathrm{~nm}$ in length, lining the lateral elongated pores. The cross strands, emanating from both sides of the slit, contacted at the slit center but had free distal endings. Shorter strands associated with the cross strands were observed at their base. Immunolabeling of recombinant nephrin molecules on transfected cells and in vitrified solution corroborated the findings in kidney. Nephrin-deficient proteinuric patients with Finnish-type congenital nephrosis and nephrin-knockout mice had only narrow filtration slits that lacked the slit diaphragm network and the 35-nm-long strands but contained shorter molecular structures. The results suggest the direct involvement of nephrin molecules in constituting the macromolecule-retaining slit diaphragm and its pores.
\end{abstract}

\section{Introduction}

Knowledge about the molecular mechanisms of plasma filtration in the renal glomeruli and mechanisms of proteinuria is still limited. The filtration barrier consists of 3 layers: a fenestrated capillary endothelium, a glomerular basement membrane (GBM), and a podocyte layer. The extracellular slit diaphragm bridges the filtration slit as a thin continuous band between podocyte foot processes covering the capillary surface in an interdigitating manner. Molecules traversing the filtration barrier are selected according to size, shape, and charge (1). Ample data suggest that the slit diaphragm forms the ultimate barrier for macromolecular permeability (2-4). Defects in the slit diaphragm lead to proteinuria, a hallmark of numerous acquired and genetic kidney diseases.

Based on EM of perfusion-fixed rodent kidneys, Rodewald and Karnovsky (5) originally proposed an isoporous zipperlike structure model for the slit diaphragm. In that model, staggered cross-bridges extend from the slit walls to a longitudinal central filament, thus forming rectangular pores in the diaphragm. This model was later questioned, particularly in light of results from freeze-etching studies with unfixed tissue using deep-etching of

Nonstandard abbreviations used: FAT, human homologue to the Drosophila tumor suppressor fat; GBM, glomerular basement membrane; NphHis, histidine-tagged recombinant nephrin; NPHS1, congenital nephrotic syndrome of the Finnish type; ZO-1, zonula occludens protein 1 .

Conflict of interest: The authors have declared that no conflict of interest exists.

Citation for this article: J. Clin. Invest. 114:1475-1483 (2004).

doi:10.1172/JCI200422562. quick-frozen samples, which suggested a sheet-like, rather than zipperlike, substructure for the diaphragm (6).

Until recently, the molecular nature of the slit diaphragm remained obscure. Nephrin (7) was the first molecule to be localized to the slit diaphragm area $(8-10)$. Nephrin is essential for the development and function of the normal glomerular filter, as seen in congenital nephrotic syndrome of the Finnish type (NPHS1), where the nephrin gene is mutated $(7,11)$. The NPHS1 disorder (12), as well as inactivation of the mouse nephrin gene (13), lead to deleterious proteinuria and absence of the slit diaphragm. For proteins of the size of albumin molecules or larger, nephrin is a decisive determinant for glomerular filtration, as seen in 3 genetic mouse models for glomerular protein leakage $(13,14)$. In addition to nephrin, P-cadherin $(15)$, the nephrin homolog Neph1 $(16,17)$, and the large cadherin-like protein FAT (human homologue to the Drosophila tumor suppressor fat) (18) have been localized extracellularly to the slit diaphragm region.

Nephrin is a type I transmembrane protein with both structural and signaling functions. Its intracellular domain is rich in serine and tyrosine residues (7) that can be phosphorylated (19). Intracellularly, nephrin apparently associates with podocin, CD2associated protein, and Neph1 (20-25). Extracellularly, nephrin molecules may interact across the filtration slit $(3,10)$. Evidence for extracellular homophilic interaction of nephrin and heterophilic interactions of nephrin and Neph1 has recently been obtained (16, 25-27). Therefore, nephrin, along with the other proteins, probably contributes to the slit diaphragm structure. 


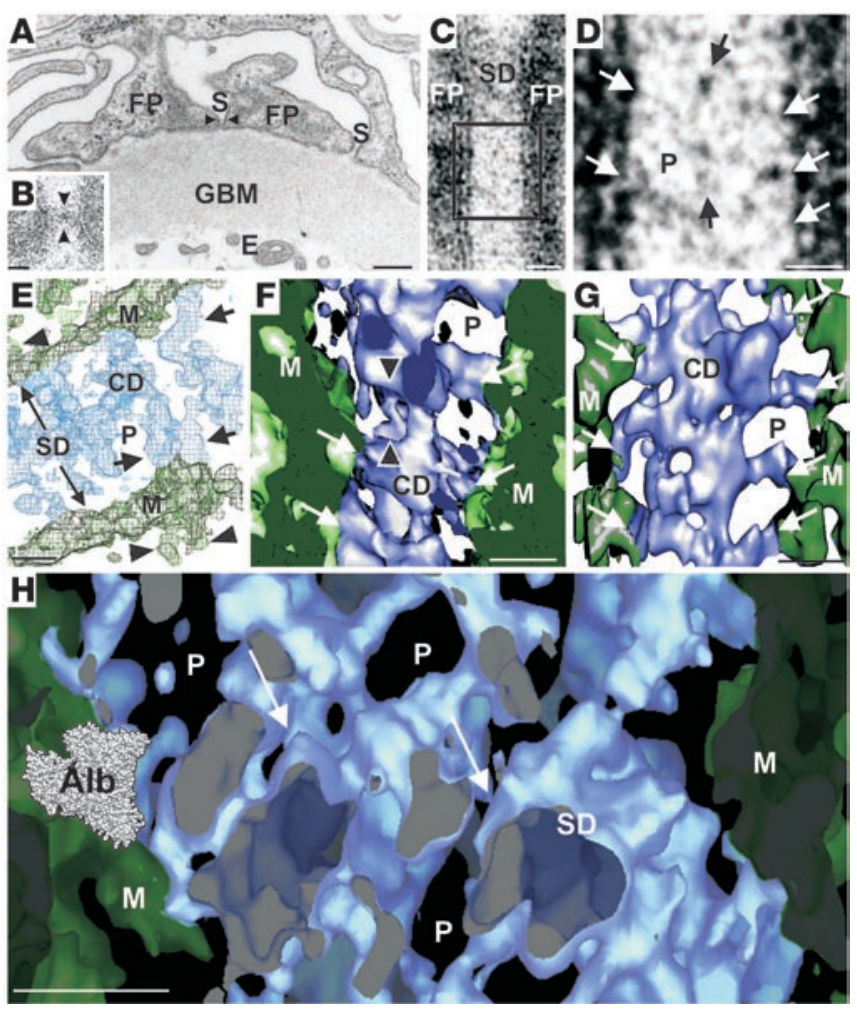

In this study, we have used electron tomography to reconstruct the $3 \mathrm{D}$ structure of the slit diaphragm, elucidate nephrin location therein, and examine slit diaphragm changes following nephrin absence. Electron tomography has provided new possibilities for visualization of cellular macromolecules and structures (28-30). It is currently the only $3 \mathrm{D}$ reconstruction method that can reveal individual cellular and molecular entities without the need for population-wide averaging. It makes imaging (31-33) and identification $(34,35)$ of individual macromolecular structures possible in their native context, usually at a resolution of up to $5-10 \mathrm{~nm}$.

Here, we present electron tomographic evidence that the slit diaphragm is a uniformly wide organized network of winding strands. The complex network contains, among shorter strands, a class of 35-nm-long cross strands, which border lateral pores smaller than albumin molecules and can be decorated with nephrin immunogold labeling. In contrast, NPHS1 patients and nephrin-knockout mice have narrow slits lacking the slit diaphragm and 35-nm strands. In addition, individual immunolabeled molecules on nephrin-transfected cells and recombinant nephrin in vitrified solution appear in electron tomography as convoluted strands, similar to those in native slit diaphragm. Based on these findings, a structural model is proposed for the slit diaphragm with a role for nephrin in constituting its porous scaffold.

\section{Results}

Electron tomography reveals a porous network of strands in the slit diaphragm. In thin-section EM of normal kidney glomeruli, podocyte foot processes were separated by an about 30- to 40-nm-wide filtration slit (Figure 1, A-D). In cross section (Figure 1, A and B), the slit dia-

Table 1

Measurements of structures in electron tomography reconstructions

\section{Sample type/structure}

Human slit diaphragm in resin section

Slit diaphragm, width

Cross strand, length

Human slit diaphragm in immuno-cryosection

Slit diaphragm, width

Nephrin-labeled cross strand, length

Cross strand, length

NPHS1 patient slit in resin section

Podocyte slit, width

Slit strand, length

Nephrin-transfected cell in resin section

Surface-strand with nephrin immunolabel, length

Recombinant nephrin in vitrified solution

Predominant class of structures, length

ANumber of reconstructions in parentheses; $\mathrm{BSD}$.

$\begin{array}{cc}\begin{array}{c}\text { Number } \\ \text { of objects }\end{array} & \begin{array}{c}\text { Measurement } \\ (\mathbf{n m})\end{array} \\ 10(7)^{\mathrm{A}} & 32 \pm 2^{\mathrm{B}} \\ 21(7) & 33 \pm 3 \\ & \\ 39(11) & 38 \pm 2 \\ 14(9) & 34 \pm 4 \\ 41(11) & 34 \pm 3 \\ 30(12) & 17 \pm 3 \\ 84(12) & 14 \pm 3 \\ 12(7) & 36 \pm 3 \\ 83(66) & 34 \pm 5 \\ \end{array}$

http://www.jci.org Volume 114 Number $10 \quad$ November 2004 


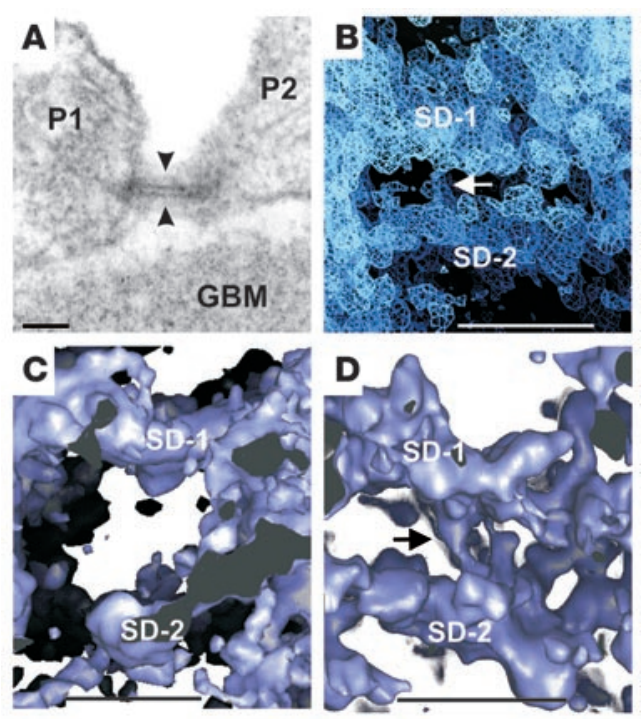

E-G). In the front view, the network for the most part had a basic zipper-type organization with staggered cross strands (Figure 1, E and $\mathrm{G}$ ) or a double ladder-type structure (Figure $1 \mathrm{~F}$ ), depending on the viewing angle and digital section thickness. On the cytoplasmic side of the slit diaphragm, strands extended internally at cross strand level (Figure 1E).

In the slit diaphragm, the cross strands merged centrally into a longitudinal density. Elongated lateral pores were found between cross strands, while smaller pores perforated the 10- to 12-nm-wide central density. This general pattern, already evident before image refinement (Supplemental Figure 1, A and B; supplemental material available at http://www.jci.org/cgi/content/full/114/10/1475/ DC1), was quite similar in all 3 species studied and in samples fixed in various ways, although ripped open areas were also found in the diaphragm. The lateral pores were in the size range of $5 \times 15 \mathrm{~nm}$, excluding constrictions (Figure 1, E-G). Several strands running at different levels in the diaphragm resulted in pores resembling winding interconnected channels with varying protrusions. Strand ends could extend into contralateral pores (Figure 1E).

Pores were also studied in mouse kidney after delicate high-pressure freezing and freeze-substitution embedding. When serum albumin volumes, rendered from crystallographic data, were superimposed on the tomograms, the larger lateral pores in the

\section{Figure 3}

Localization of nephrin in human slit diaphragm: immuno-cryolabeling of extracellular terminal Ig-domains of nephrin. SD pores $(P)$ are indicated. Scale bars: $40 \mathrm{~nm}$ (A and B), $10 \mathrm{~nm}$ (C), $5 \mathrm{~nm}$ (D). (A) Nephrin label (5-nm gold, arrows) in EM along obliquely cut slit diaphragm. (B) Tomogram of filtration slit bordered by GBM, foot processes, and slit diaphragm. Gold labeling for nephrin appears under the diaphragm at different levels of the digital volume. Sigma levels: 0.05 (tissue), 13 (gold particles). (C) Higher magnification of B (same sigma levels), but visualized from below, through 30-nm-thick digital section encompassing the slit diaphragm. Around 4-nm-wide strands (arrows) extend from the podocyte surface into the diaphragm. (D) Close-up of slit diaphragm cross strand (arrow). Gold label (G) appears near the distal end of cross strand. Note associated globules (sectioned short strands) at the proximal part of cross strand. Only small volume differences in wire frames exist between sigma levels 1.0 (blue and green) and 0.3 (white).

\section{Figure 2}

Human, rat, and mouse slit diaphragm with double layers. Scale bars: $50 \mathrm{~nm}$ (A), $20 \mathrm{~nm}$ (B-D). (A) Filtration slit in EM cross section between foot processes of human podocytes (P1, P2) showing double-layered slit diaphragm (arrowheads). Tannic acid-glutaraldehyde and osmium fixation; resin section. (B) Tomogram of same slit as in A. Strands seem to connect the two slit diaphragm layers (arrow). Sigma level: 0.5. (C) Same rat filtration slit as in Figure $1 \mathrm{~F}$, tilted $90^{\circ}$ around the $x$ axis; showing 2 layers in slit diaphragm. Sigma level: 1.0. (D) Two layers in crosscut mouse slit diaphragm with connecting strands (arrow); glutaraldehyde and osmium fixation. Sigma level: 1.0.

diaphragm were seen to be of similar or smaller size compared with albumin molecules (Figure 1H). Smaller pores were found between strands in the central density in all 3 species (Figure 1, E-H).

Measured from tomogram reconstructions, the glutaraldehyde-fixed human slit diaphragm in conventional resin sections was about 30-35 nm wide (Figure 1E and Table 1). Rat and mouse diaphragms were found to be of nearly similar width. Slit widths varied somewhat depending on the fixation and embedding techniques used. Also, locally, the widths fluctuated slightly in all 3 species, which resulted from undulations of the slit wall (Figure 1, E-H). With high-pressure freezing and freeze-substitution embedding of immersion-fixed mouse kidney, the slit width was around $40 \mathrm{~nm}$ (Figure 1H). The human slit diaphragm was also about $40 \mathrm{~nm}$ wide in cryosections (Table 1), but sometimes more distended slits were found.

The thickness of the slit diaphragm layer for the most part varied between 5 and $10 \mathrm{~nm}$, with several strands often contributing to its thickness (Figure $1 \mathrm{~F}$ and Figure 2). In addition, separate layers of slit diaphragm were occasionally observed in all 3 species, and interconnecting strands were found between layers (Figure 2, B and D).

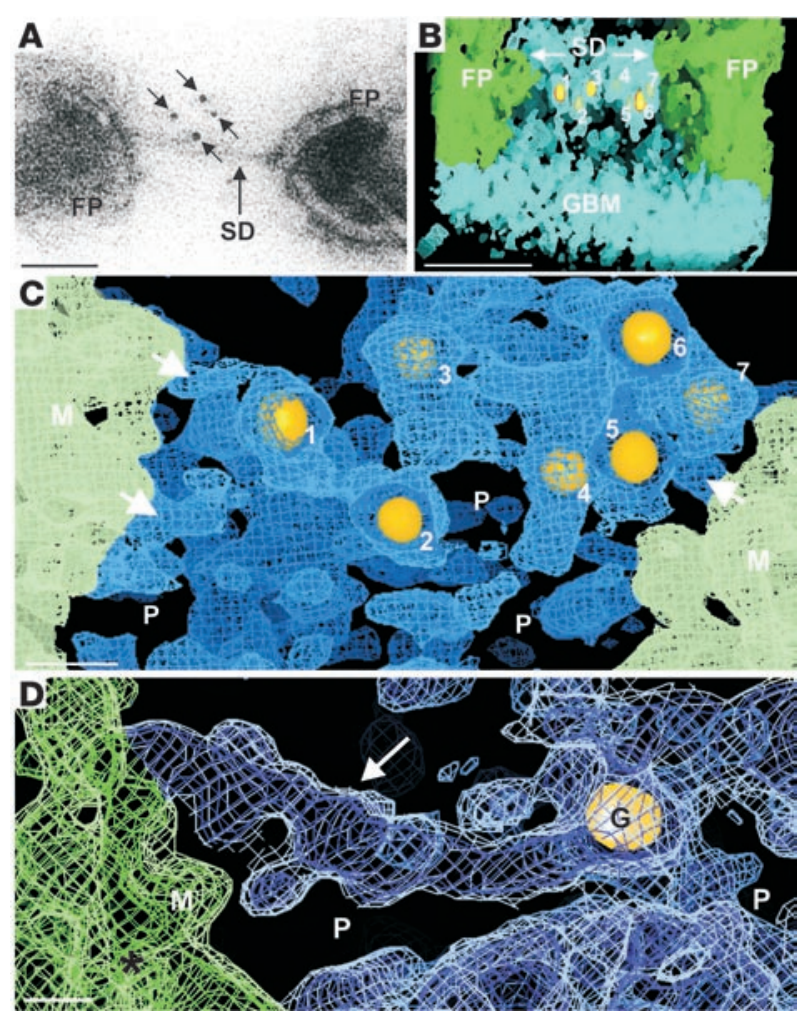




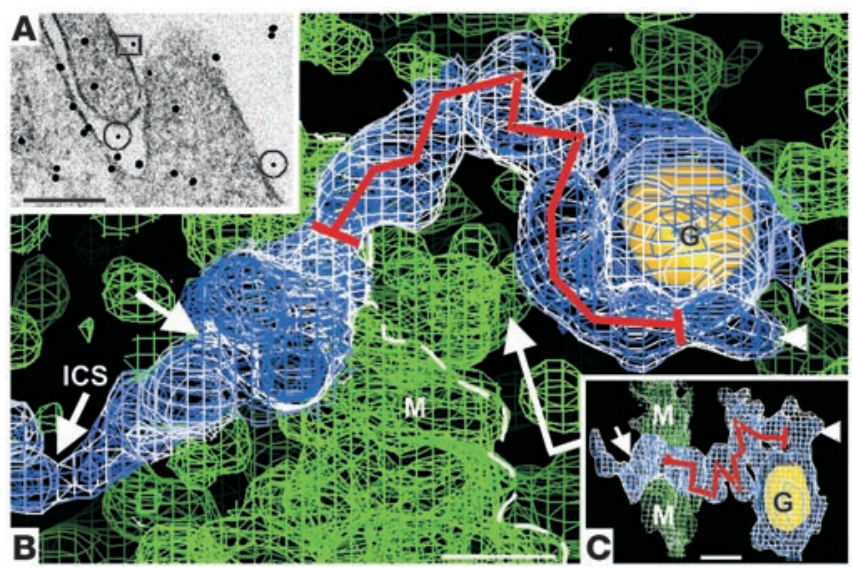

Nephrin is a structural component of the slit diaphragm. We then studied the location and orientation of nephrin molecules in the native slit diaphragm. In immunolabeling of EM cryosections, the N-terminus of human nephrin was found extracellularly in the diaphragm area (Figure 3A), as previously demonstrated in immunoresin sections (10). In the tomograms, the gold label for the $\mathrm{N}$-terminal end of nephrin could be localized widely across the diaphragm (Figure 3, B and C). In certain samples, individual convoluted cross strands extended from the plasma membrane into the diaphragm, especially at its lower border facing the GBM, with the gold marker always associated with the distal end of the strand (Figure 3D). The length of the labeled strands (about $35 \mathrm{~nm}$; Table 1) was most often difficult or impossible to determine due to the complexity of the strand-antibody-immunogold conglomerate and the diaphragm network. In the proximal part of the labeled strands, associated shorter globular strands were found (Figure $3 \mathrm{D})$, best visible in stereo viewing of thicker digital sections.

Expression and structure of recombinant nephrin in transfected cells. To better confirm the identity of the immunolabeled molecules observed in native slit diaphragm, we transfected full-length nephrin to HEK293 cells, which normally do not express this protein (27), and stained for nephrin by pre-embedding immunogold labeling. In EM, surface-restricted nephrin label was found on and between cells (Figure 4A). Tomograms of such anti-neph-

\section{Figure 5}

Recombinant nephrin and IgG in vitrified solution in electron tomography. Scale bars: $5 \mathrm{~nm}$. (A) Schematic domain structure of nephrin. C, intracellular carboxyl terminal; filled rectangle, transmembrane domain; hexagon, fibronectin type III-like domain; incomplete circles, Ig repeats with internal disulfide bonds (dashes); S-shaped line, flexible region; $\mathrm{N}$, extracellular amino terminal; $\mathrm{SH}$, regions with free cysteine residues. (B) Schematic drawing of soluble NphHis. Ig domains 1 and 8 and fibronectin type III-like domain (Fn III) are indicated. (C) Tomogram of vitrified solution of recombinant NphHis: solitary (1) and associated (2) structures. Sigma levels: 2.0 (orange) and 1.5 (blue). (D and $\mathbf{E}$ ) Structures in solution of NphHis and anti-nephrin IgG (against $\lg -1$ and Ig-2 repeats of nephrin). Y-shaped structures (putative IgG) are associated with the last 2 globules in the long arm of the strands. Arrow indicates putative flexible region of NphHis. Density refinement to a resolution of $25 \AA$. (F) Tomogram of individual reference IgG molecule. Putative Fc region points downward. Refinement and low-pass filtering to $25 \AA$. Volume rendering with contouring levels corresponding to sigma levels 1.5 in $\mathbf{D}$ and $\mathbf{F}$ and 2.5 in $\mathbf{E}$.

\section{Figure 4}

Extracellular nephrin-label on transfected HEK293 cells. Scale bars: 100 $\mathrm{nm}$ (A), $5 \mathrm{~nm}$ (B and C). (A) Small, 5-nm-immunogold particles (in the rectangle and 2 circles) mark nephrin on the cell surface. (The large 10-nm-gold particles are used as coordinates for 3D-reconstruction purposes $[39,67]$.) Pre-embedding immunolabeling; resin section. (B) Tomogram from reconstructed volume of rectangle in $\mathbf{A}$. The strand with a gold label on its distal end seemingly traverses the cell membrane. Marked extracellular length, measured in 3D, is about $35 \mathrm{~nm}$ when the putative anti-nephrin IgG complex (5-nm-gold-anti-rabbit IgG + rabbit anti-nephrin $\lg G$ ) at the end of the strand (arrowhead) is omitted. Inside the cell, the strand is continuous near the membrane (short arrow) with intracellular strand (ICS). Sigma levels: 0.5 (green and blue) and 0 (white, strand-immunogold complex); 13 (gold particle). (C) A $90^{\circ}$-tilted side view from the direction shown in B (long, bent arrow). Sigma levels: 0.3 (green and blue) and 0 (white). Note minimal volume change in strand between sigma levels in $\mathbf{B}$ (from 0.5 to 0 ) and $\mathbf{C}$ (from 0.3 to 0 ).

rin-immunogold complexes on the cell surface membrane (rectangle in Figure 4A) showed strands with a globular substructure, associated at their distal end with the gold label (Figure 4, B and C). The approximate length of the labeled extracellular strands agreed with that of the measurable labeled cross strands in native slit diaphragm (Table 1).

Electron tomographic analysis of individual recombinant nephrin molecules in vitrified solution. Next, we studied individual extracellular nephrin molecules in vitrified solution for comparison with the convoluted 35-nm strands observed in the slit diaphragm and on transfected cells. To this end, a soluble histidine-tagged recombinant nephrin (NphHis) was expressed in HEK293 cells, purified, and analyzed by electron tomography. Compared with full-length nephrin (Figure 5A), NphHis only contained the extracellular domain linked to a histidine tag (Figure 5B). Tomograms of NphHis molecules in vitrified solution revealed twisted strands with globular substructures (Figure 5C). The strands usually formed
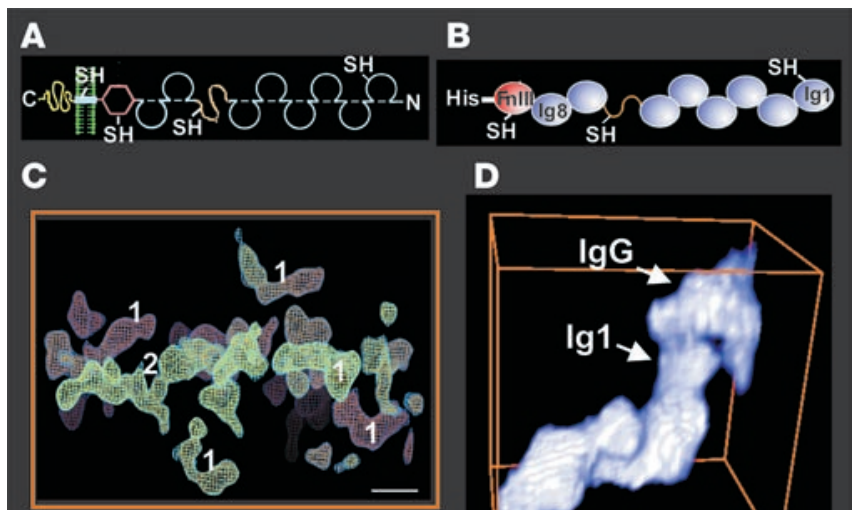

D

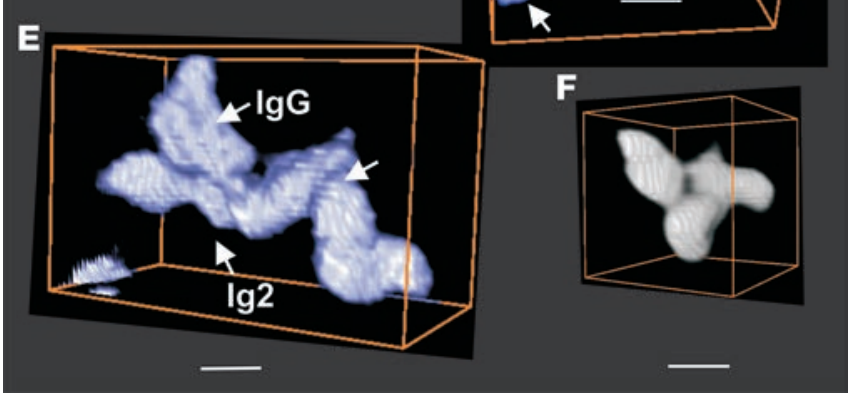




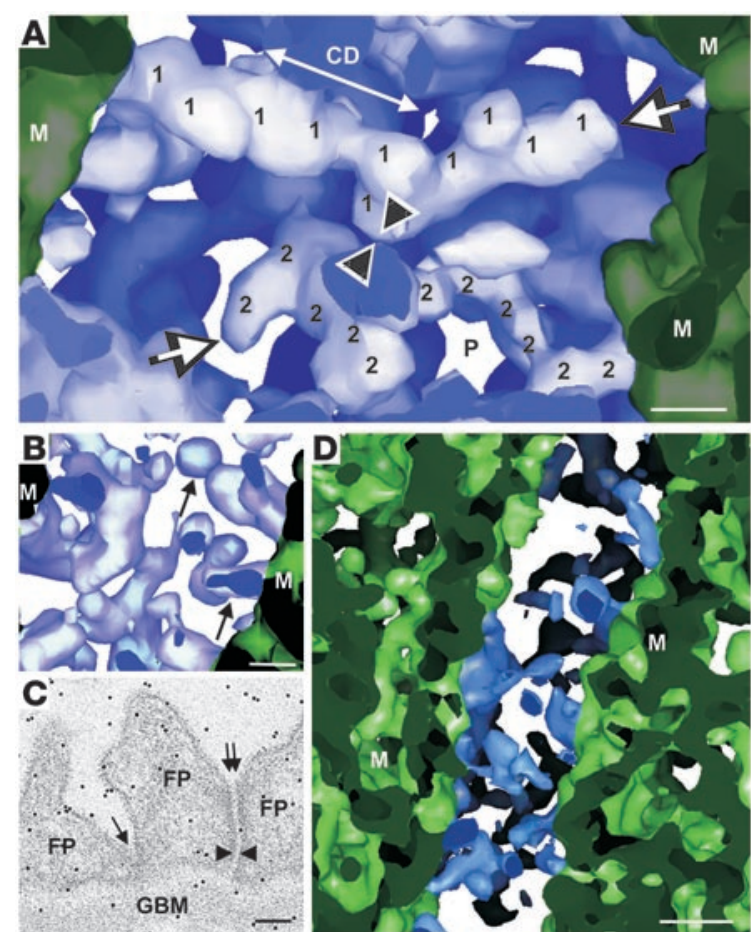

loops or back-folds. Most of the strands fell into a class of about 35 $\mathrm{nm}$ in length (Table 1). Dimer-type connected strands and smaller structures were also present (Figure 5C).

Identification of strands was attempted by mixing $\mathrm{NphHis}$ before freezing with IgG directed against the first two Ig-like domains of nephrin (Figure 5B). When present, a spread, Y-shaped particle, corresponding to the typical IgG structure (Figure 5F), was associated with the first or second globule of a 35-nm strand (Figure 5, D and E). In addition, strand doublets linked at one end by a Y-shaped particle were found (data not shown). The extended strandlike 35-nm structures closely resembled the convoluted 35-nm strands observed in native slit diaphragms and on transfected cells.

To estimate the resolution obtainable in tomograms of vitrified structures, we studied a purified reference IgG sample (see Methods). The broad Y shape of the IgG with its 3 lobes was well recognizable in the structures found (Figure 5F). These tomograms of IgG compared favorably with densities obtained by x-ray crystallography of IgG2a at 4-nm resolution (37) and came close to the 3-nm resolution previously obtained in electron tomograms $(38,39)$.

Strand substructure and contacts in native slit diaphragm. Tomograms of tissues, fixed by immersion in a mixture of tannic acid and glutaraldehyde, revealed globular strand substructures and close association between cross strands in the central slit diaphragm (Figure 6A). Only a class of about 35-nm-long cross strands could clearly be established within the diaphragm (Figure 6A and Table 1). Lack of resolution and intertwining of strands made most of the strands impossible or difficult to delineate and measure, especially in the central part of the slit diaphragm. In favorable cases, 35-nm strands extending over the slit center were seen to have a handleshaped ending coming close to, but not contacting, the opposite podocyte cell membrane. In tomograms with the best resolution, these approximately 3- to 6-nm-wide strands had a discernible globular substructure (Figure 6A), first discernible after image

\section{Figure 6}

Comparison of normal and nephrin-deficient glomerular filtration slits. Fixations: tannic acid-glutaraldehyde immersion in $\mathbf{A}$ and $\mathbf{B}$ and glutaraldehyde and osmium in $\mathbf{C}$ and $\mathbf{D}$; resin sections. Scale bars: $5 \mathrm{~nm}$ (A, B, and D), $100 \mathrm{~nm}$ (C). (A) Tomogram of human filtration slit, front view; thick digital section. Globular cross strands, about $35 \mathrm{~nm}$ in length (labeled 1 and 2), extend from podocyte membranes. Arrows indicate distal strand ends close to, but not contacting, the opposite membrane. Arrowheads indicate close association of strands at the central density. Contacting short strand at base of cross strand 1. Pore openings are indicated. Surface rendering; sigma level: 0.1. (B) Human podocyte slit, digital section right below the slit diaphragm above the GBM. Short strands (arrows) with up to 5 globules stretch into a narrow region of the filtration slit. This stretching is also sometimes seen in EM. Sigma level: 0.1. (C) Sample from NPHS1 patient 1, homozygous for Fin-major mutation of nephrin; EM of narrow cross-cut filtration slits. Shorter (arrow) or longer (double arrow) stretches of contact are shown. The EM image was taken after a low-dose tilt series and still shows no sign of section contamination. Slit width at arrowheads is about $10 \mathrm{~nm}$. (D) Sample from NPHS1 patient 2, Fin-major homozygote; tomogram of cross-cut slit slightly above GBM. Only short globular strands are now seen between cell membranes in the narrow (about 10-15 nm) filtration slit. Surface rendering; sigma level: 0.2.

refinement (Supplemental Figure 1, C and D). Overlaps and twists could increase the apparent strand width up to $10 \mathrm{~nm}$ (see also Figure 1, E-H). The spacing of the cross strands at their membrane origin was mostly 10-12 nm. However, the distance seemed to vary somewhat, depending on the thickness of digital sections, as the cross strands were not all in the same plane and occasionally ran parallel to each other (Figure 1, E-G).

Other types of strands, half the length or less of the 35-nm cross strands, were found on the cell membrane close to and outside the slit diaphragm level. Such strands were also seen in the narrow contact sites occasionally found between the podocyte foot processes (Figure 6B). In the diaphragm, the short strands seemed to be in contact with cross strands at their basal end (Figure 6A).

Podocyte filtration slits in NPHS1 patients and nephrin-deficient mice. Kidneys from NPHS1 patients with massive proteinuria, podocyte effacement, and absence of slit diaphragm as shown by EM (12) were then studied by electron tomography. In samples from NPHS1 patients, the podocyte slits were typically narrow (about $15-20 \mathrm{~nm}$, but occasionally over $50 \mathrm{~nm}$ in width; Table 1), and they lacked the slit diaphragm (Figure 6C). The organized diaphragm network with its 35-nm-strands, found in normal human slits (Figure 6A), was absent in NPHS1 kidneys (Figure 6D). Instead, the slit contained mostly shorter, bent strands along a cell-contact zone running parallel but occasionally also perpendicularly to the GBM (Figure 6C). The strands (15 $\mathrm{nm}$ in average length; Table 1) consisted of up to five about 3- to 4-nm-wide globular subdomains (Figure 6D). The short strands were partially in contact with similar strands across the slit.

Likewise, in the nephrin-knockout mice, mostly narrow podocyte filtration slits were found, as well as the total absence of slit diaphragm, as previously reported (13). As in tomograms of NPHS1 patients (Figure 6D), short molecular strands, partly bridging the narrow podocyte slits, replaced the slit diaphragm network in the knockout mice (data not shown). 


\section{Discussion}

Architecture of the slit diaphragm. The present work provides new insight into the $3 \mathrm{D}$ molecular structure of the podocyte slit diaphragm by the use of electron tomography. Analyses of glomeruli from 3 mammalian species demonstrated that the basic architecture of the slit diaphragm is an organized structure with a network of strands forming somewhat irregular elongated pores on both sides of a central, denser region. These $3 \mathrm{D}$-reconstruction results were obtained from studies of sections of heat-cured resin with well-fixed samples, as well as from thawed frozen sections of lightly fixed material. Importantly, the findings were also confirmed by using the combination of the high-pressure freezing and freezesubstitution embedding procedures, which is considered to be one of the most reliable tissue preparation methods for ultrastructural preservation (40). The results described here appear, therefore, to be both consistent and repeatable.

A deeper analysis of pore sizes was not found to be feasible because of the high variability in pore shape. However, comparison with superimposed albumin molecules, rendered from $x$-ray crystallographic data, showed that the pore size of the larger, lateral pores was similar to, or smaller than, albumin molecules. As the pore walls were formed by bent cross strands running at several levels in the slit diaphragm and the strands butted into pores on the contralateral side, the pores appeared more like convoluted interconnected channels with varying diameter rather than simple straight holes of a regular size. It would obviously be difficult for molecules of the size of albumin (about $80 \times 80 \times 30 \AA$; refs. 41 and $42)$ to pass through these pores. Consequently, the slit diaphragm is likely to contribute to the size-selective barrier of the glomerular filter. This notion is further corroborated by the observed absence of a slit diaphragm network in tomograms from the glomeruli of NPHS1 patients and nephrin-knockout mice with heavy proteinuria. However, while the small pores or channels of the slit diaphragm can provide a physical barrier for albumin and larger plasma proteins, the existence of a charge barrier (1) or a gel exclusion effect (43) might be necessary to prevent clogging of the filter.

An interesting side finding that was verified in electron tomography was the occasional local stratification of the slit diaphragm into 2 or more layers. Although observable in some previously published EM images, this characteristic has not gained much attention. It is tempting to speculate that this could be associated with a turnover or repair process of the slit diaphragm in the adult kidney. The stacked bands (ladderlike structures) seen in cross-cut junctions between differentiating podocytes in embryonic kidneys $(44,45)$ and in disease and experimental states $(46,47)$ might be redundant slit diaphragm layers, as previously suggested (46). This is also supported by the presence of zonula occludens protein 1 (ZO-1) on the cytoplasmic side of ladder-like structures in crosscut podocyte filtration slits in developing human glomeruli (44). However, it has also been reported that in experimental rat nephrosis, ZO-1 is absent from the visible ladderlike structures but present in newly forming occluding-type junctions and remaining slit diaphragms (48). The ladderlike structures seen in cross-sectioned embryonic or perturbed podocyte contacts are not to be confused with the ladderlike stretches described in tangential sections of slit diaphragm of normal adult glomeruli, discussed below.

In general, our results agree quite well with the slit diaphragm structure model previously put forward by Karnovsky and coworkers $(5,49,50)$. The model was based on their studies on resin sections and freeze-etching replicas of fixed rodent and human kidneys. They proposed that in parallel view, the slit diaphragm - found to be a continuous $2 \mathrm{D}$ band extending between all podocyte foot processes - has a zipperlike isoporous structure with an average pore size of $40 \times 140 \AA$ in the rat, that is, in the same size range as found in the present study. Later, Hora et al. (6) described 3 substructure types in the rat slit diaphragm. In metal replicas, tannic acid-glutaraldehyde-fixed slit diaphragm had sheet-, ladder-, and zipperlike stretches, whereas unfixed quick-frozen and deep-etched tissues mostly displayed sheet-like diaphragm areas. Slit diaphragms, heavily fixed with glutaraldehyde and osmium, again, had zipperlike substructures. It was concluded that fixation artifacts transformed the native sheet-like substructure into the other forms. In addition, shrinkage of foot processes seen after chemical fixation, but not after fixation by quick-freezing-freeze-substitution, was proposed to cause the concurrent increase noticed in slit width (51).

Our fixation conditions did not, however, result in cellular shrinkage and widening of the slit diaphragm (see Table 1). Also, it must be emphasized that our results with the combination of the high-pressure freezing and freeze-substitution embedding approaches were consistent with results we obtained with the other methods. As to the freeze-etching results of Hora and coworkers (6), a slightly oblique deep-etched plane running above, through, and under a thin slit diaphragm layer could well explain the consecutive different structural forms along the podocyte contacts. This could also explain why the sheet-like stretches were found to be narrower than the ladderlike diaphragm areas.

Components of the slit diaphragm. The present work provides the first direct evidence for involvement of the extracellular domain of nephrin in the slit diaphragm structure. Nephrin has previously been localized by immuno-EM to the slit-diaphragm region of human and rodent kidneys (8-10). This was also shown in the present study by examination of immunolabeled cryosections. Although they produced the best labeling intensity, maybe as a result of better antibody/gold label accessibility, this was probably more as a result of an understaining of nephrin epitopes. The result, however, clearly demonstrated nephrin involvement in the slit-diaphragm structure. Our electron tomography findings of strand contacts in the diaphragm support, but do not prove, that extracellular domains of nephrin molecules interact in the slit diaphragm with each other and with other molecules, thus maintaining a certain, maybe flexible slit width of around 30-45 nm. However, the lack of slit diaphragm and presence of only narrow podocyte slits in NPHS1 patients and nephrin-knockout mice suggest that nephrin molecules contribute to the building and maintenance of a filtration slit of normal width and diaphragm structure.

Direct evidence for homophilic interactions of nephrin comes from results with soluble extracellular domains of nephrin using surface plasmon resonance (27). In addition, other recent studies have provided data consistent with this idea $(25,26)$. The observed complexity of the slit diaphragm and methodological questions concerning fixation, embedding, immunolabeling, contrast staining, and electron tomography resolution still leave open the detailed organization and molecular interactions of nephrin in the diaphragm. However, it was obvious that nephrin-immunolabeled strands (Figure 3, C and D) as well as nephrin-like 35-nm-strands (Figure 6A) lined the pores of the diaphragm and apparently were in contact, most plainly at the center of the diaphragm, with other similar strands from the opposite slit side. Interestingly, strand dimers were also present in the vitrified NphHis solution. 
Besides nephrin, additional molecules, not studied here, may also contribute to the formation and maintenance of the slit diaphragm. In immuno-EM, P-cadherin (15) and FAT (18) have been localized in podocytes close to the diaphragm area. However, the P-cadherin expression pattern differs developmentally from that of nephrin (44), and P-cadherin does not immunoprecipitate with nephrin or Neph1 (17). Also, in kidneys of NPHS1 fetuses, which lack slit diaphragm and nephrin, P-cadherin is expressed normally (44). Furthermore, since P-cadherin-deficient mice exhibit no kidney abnormalities or function (52), the protein presumably is not essential for the slit diaphragm. A mouse knockout of the gene for FAT, the over-500-kDa protein with cadherin-like extracellular repeats, resulted in neonatal lethality but of unknown cause (53). The FAT family members may, however, have a sensing role rather than participating in cell adhesion (54). In addition, mice deficient for the nephrin-related transmembrane protein Neph1 with extraand intracellular nephrin association $(17,25,26)$ exhibit proteinuria and die at 2-3 weeks of age (16).

At present, it has not yet been shown by electron tomography where these other proteins reside in the slit diaphragm network or elsewhere in the filtration slit. The nature of the shorter molecular strands with 5 or fewer globular subunits seen in tomograms associated with the proximal part of the $35-\mathrm{nm}$ cross strands or near the diaphragm is yet not known. The same applies to the short strands between podocytes in NPHS1 patients and nephrinknockout mice. Both cadherins $(55)$ and Neph1 $(16,56)$ are known to be composed of 5 extracellular subdomains.

In the tomograms, the labeled nephrin strands on transfected cells and in the human slit diaphragm seemed to be continuous with intracellular molecular strands. In addition to Neph1, other molecules interacting intracellularly with nephrin have been reported. These include CD2AP, an actin-binding protein with a kidney phenotype in knockout mice (21). Likewise, an integral membrane protein podocin (NPHS2) may associate nephrin with the actin network as it facilitates nephrin signaling (19) and interacts with Neph1 (56). In addition, mutated podocin leads to congenital nephrotic syndrome (57). ZO-1, a component of the cytoplasmic face of slit diaphragm $(58,59)$, could also be involved in connecting the diaphragm to the podocyte cytoskeleton, as direct interaction between ZO-1 and Neph1 has been found $(17,60)$. Interestingly, nephrin oligomeres have been shown to associate with plasma membrane lipid rafts containing a podocyte specific GD3 ganglioside (61).

Strands on transfected cells and in solution. Electron tomographic analysis of the nephrin-immunolabeled strands present on the surface of nephrin-transfected HEK293 cells showed that these 35-nm-long molecules have an elongated twisted form. Like the cross strands in the native slit diaphragm, the extracellular strand portion seemed to cross the cytoplasmic membrane and have an intracellular continuation. The globular substructures of the strands can be attributed to the Ig domains and to the fibronectin type III-like motif of nephrin (Figure 5B; ref. 10), the latter localized at the end of the short arm (membrane-end) of the recombinant NphHis variant. The structural similarity of the nephrin-immunolabeled strands in the slit diaphragm, in transfected cells, and in vitrified solution is quite clear, and this speaks strongly in favor of the $35-\mathrm{nm}$ strands in the native diaphragm truly being extracellular portions of nephrin molecules. Model for the slit diaphragm and mechanisms of proteinuria in NPHS1 patients. Based on the present results, we propose a nephrin-containing network model for the structure of the podocyte slit diaphragm. It takes into consideration the localization of nephrin in
immuno-EM, the structural characteristics of the molecule, and the novel electron tomography findings presented in this work. This model is partly a molecular-level version of the Rodewald and Karnovsky zipper model (5), where nephrin strands from the 2 opposing slit walls cross the slit diaphragm and form filtration channels, which are to small for albumin-sized molecules to pass through them. Molecular interactions may exist between the nephrin strands, most notably at the slit center. Other slit diaphragm proteins that have not yet been localized in detail by electron tomography may further contribute to the network. The model also allows for a dynamic nature of the slit diaphragm $(61,62)$. Changes in the dimensions of the diaphragm and its pores could take place by stretching or retraction of the coiled and bent cross strands and may explain, in part, the variations seen by different methods in slit diaphragm width and appearance.

The present study provides new insight into the mechanisms of proteinuria that occurs in NPHS1 patients. Thus, the loss of nephrin causes disappearance of the uniformly wide porous slit diaphragm that functions as the kidney macromolecular ultrafilter, and thereby causes proteinuria. In that case, the slit is narrow and lacks an ordered filter structure, which provides practically free passage for plasma proteins through the slit. It would be interesting to use electron tomography to study the $3 \mathrm{D}$ structure of the slit diaphragm in proteinuria cases caused by mutations in the genes for other slit diaphragm proteins or in proteinuria due to unknown causes.

\section{Methods}

Samples and procedures for EM and electron tomography. Human kidney samples were obtained from healthy individuals (10) and 2 NPHS1 patients homozygous for the Fin-major mutation not expressing nephrin protein (12). Samples from kidneys of Sprague-Dawley rats were collected and studied with the approval of the ethical commission and human samples collected and studied with the approval of the Ethical Committee of the Diseases and Psychiatry of Children and Adolescents at the University of Helsinki. The kidneys from C57BL/6 and 129/SV (WT and nephrin-knockout) mice (13) were collected and studied with the approval of the North Stockholm Ethics Committee for Animal Experiments at the Karolinska Institutet.

Sections for EM were obtained from tissue samples in epoxy resin (LX-112) using standard fixation, embedding, and preparation methods (10). Immersion fixation with $2.5 \%$ glutaraldehyde in $0.1 \mathrm{M}$ phosphate buffer with or without postfixation in $1 \% \mathrm{OsO}_{4}$ or $1 \%$ tannic acid- $1 \%$ glutaraldehyde and osmium was employed. Alternatively, perfusion fixation with glutaraldehyde (perfusion pressure $150 \mathrm{~cm} \mathrm{H}_{2} \mathrm{O}$ ) was used with rat and mouse kidneys.

For immuno-EM, the samples were fixed with $3.5 \%$ paraformaldehyde alone or with different concentrations $(0.01-0.1 \%)$ of glutaraldehyde and stained as described $(10,44)$. In short, sections of samples embedded in LR White resin (Electron Microscopy Sciences) were treated with primary antibodies and 5- or 10-nm gold-conjugated secondary antibodies and post-stained in $1 \%$ uranyl acetate. Nonimmune rabbit IgG was used as the primary antibody for controls. Immuno-cryosections, produced by the modified Tokuyasu thawed frozen sectioning technique (63), were immunostained as above.

For electron tomography, normal epoxy resin, immunoresin, and immunocryosections were prepared as for EM. In addition, for high-pressure freezing and freeze-substitution embedding, pieces of mouse kidney cortex $\left(<1 \mathrm{~mm}^{3}\right.$, unfixed or fixed in $2.5 \%$ glutaraldehyde) were placed between 2 flat aluminum specimen holders and high-pressure frozen in a Balzers HPM 010 device (Bal-Tec AG). The frozen specimens were stored in liquid nitrogen before use. 
Subsequently, the specimens were freeze-substituted in a Leica AFS apparatus (Leica Microscopy and Scientific Instruments Group). The protocol involved fixation in methanol containing $1 \% \mathrm{OsO}_{4}$ for 10 hours at $-95^{\circ} \mathrm{C}, 8$ hours at $-62^{\circ} \mathrm{C}$, and 3 hours at $-40^{\circ} \mathrm{C}$. The specimens were warmed to room temperature, rinsed in acetone, and embedded in LX-112 (Ladd Research). In electron tomography immunolabeling, only $5 \mathrm{~nm}$ immunogold was used, and sections were finally overlaid with 10-nm gold-protein A particles (Amersham Biosciences) for alignment purposes. Cultured cells for immuno-EM and electron tomography were prefixed with light glutaraldehyde and immunogoldlabeled before postfixation and embedding in epoxy resin.

For vitrified electron tomography samples, recombinant nephrin (1 mg/ $\mathrm{ml}$ of protein in $20 \mathrm{mM}$ Tris- $\mathrm{HCl}$ with $1 \mathrm{mM} \mathrm{CaCl}_{2}$ ) was mixed with $10-\mathrm{nm}$ gold-protein A solution (Amersham Biosciences), placed on grids coated with perforated carbon, and rapidly frozen in liquid ethane at liquid nitrogen temperature (38). For immunolabeling, $\mathrm{NphHis}$ nephrin was mixed with anti-nephrin antibody solution before vitrification. The reference IgG, mAb 250 (64), a kind gift of A.-C. Wikström and Örjan Wrange (Karolinska Institutet), was vitrified in a manner similar to recombinant nephrin.

Antibodies. Purified polyclonal rabbit anti-human nephrin antibodies against the $\mathrm{N}$-terminal extracellular domains Ig1 and Ig2 (AA 22-240) of human nephrin were used $(10,44)$.

EM and electron tomography. Transmission EM was used as previously described (10). Electron tomography was performed essentially as published earlier $(38,65,66)$ using a Philips CM200 field-emission gun transmission electron microscope. Automatic tilt series of low-dose images were taken with a slow-scan camera $(2048 \times 2048$ CCD chip, pixel size 14 $\mu \mathrm{m})$ using the TVIPS $\mathrm{GmbH}$ low-dose data collecting system. Images were recorded at $1^{\circ}$ or $2^{\circ}$ tilt intervals $\left(-65\right.$ to $+60^{\circ}, \times 26715$, final pixel size of $5.24 \AA)$. The total dose on the cryosamples was $15-20 \mathrm{e}^{-} / \AA^{2}$. With immunocryosections, it was $20-40 \mathrm{e}^{-} / \AA^{2}$ and with resin sections, below $50 \mathrm{e}^{-} / \AA^{2}$.

Geometrical image alignment was performed using gold markers (error usually under 1 pixel, $5.24 \AA$ ). Density refinement was done using the program COMET (39). This resulted in vitrified samples with an end resolution below $5 \mathrm{~nm}$, as based on previous results in which the $\mathrm{x}$-ray crystal structure of IgG was docked into tomograms $(38,66)$. The reconstructions were visualized by isodensity contouring as surface rendered or wire-frame representation using the program XTV (67) or by volume rendering with the program BOB (provided by Ken Chin-Purcell, Minnesota Supercomputer Center Inc.). To superimpose human serum albumin for reference into tomograms, a space-filled model of the albumin crystal structure (41) was rendered using Visual Molecular Dynamics visualization software (68).
Nephrin variants and cell transfection. The cDNA clone (pcDNA3NPH1) encoding full-length human nephrin has been described (69). A cDNA clone encoding the soluble histidine-tagged nephrin (pCR3Nph-His) was generated from the full-length clone. A Sall restriction site (GTCGAC) encoding amino acid residues Val and Asp was introduced in frame after the sequence encoding the last 4 amino acid residues (GlyPro-Ser-Gly) of the fibronectin type III-like domain of the nephrin sequence. A short, $3^{\prime}$-fragment encoding 6 histidine residues followed by a stop codon was introduced into this Sall-site. The final construct encoded a soluble NphHis molecule with the entire extracellular portion of nephrin followed by 2 additional residues (Val-Asp) and a histidinetag (-Gly-Pro-Ser-Gly-Val-Asp-His 6 ). The cell lines and purification of recombinant nephrin have been reported $(27,69)$.

\section{Acknowledgments}

We thank the technical staff of the Electron Microscopy Unit, Institute of Biotechnology, University of Helsinki, and are grateful to Lars Haag for technical help in high-pressure freezing and freezesubstitution embedding. Granting agents include Sidec Technologies AB, The Agouron Institute, The Swedish Foundation for Strategic Research, The Swedish Research Council, The Knut and Alice Wallenberg Foundation, The Foundation of Knowledge and Competence Development, The Sigrid Jusélius Foundation, The Novo Nordisk Foundation, NIH grant DK-54724, and European Union grants BIO4-CT97-2364 and BIO4-CT96-0099.

Received for publication June 29, 2004, and accepted in revised form August 17, 2004.

Address correspondence to: Karl Tryggvason, Department of Medical Biochemistry and Biophysics, Karolinska Institutet, SE 171 77 Stockholm, Sweden. Phone: 46-8-7287720; Fax: 46-8-316165; E-mail: karl.tryggvason@mbb.ki.se.

Jamshid Khoshnoodi's present address is: Department of Medicine, Division of Nephrology and Hypertension, Vanderbilt University Medical Center, Nashville, Tennessee, USA.

Jingjing Zhang's present address is: Renal Division, Department of Medicine, Brigham and Women's Hospital and Harvard Medical School, Boston, Massachusetts, USA.
1. Kanwar, Y.S., Liu, Z.Z., Kahihara, N., and Wallner, E.I. 1991. Current status of the structural and functional basis of glomerular filtration and proteinuria. Semin. Nephrol. 11:390-413.

2. Endlich, K., Kriz, W., and Witzgall, R. 2001. Update in podocyte biology. Curr. Opin. Nephrol. Hypertens. 10:331-340

3. Tryggvason, K., and Wartiovaara, J. 2001. Molecular basis of glomerular permselectivity. Curr. Opin. Nephrol. Hypertens. 10:543-549.

4. Mundel, P., and Shankland, S.J. 2002. Podocyte biology and response to injury. J. Am. Soc. Nephrol. 13:3005-3015.

5. Rodewald, R., and Karnovsky, M.J. 1974. Porous substructure of the glomerular slit diaphragm in the rat and mouse. J. Cell Biol. 60:423-433.

6. Hora, K., Ohno, S., Oguchi, H., Furukawa, T., and Furuta, S. 1990. Three-dimensional study of glomerular slit diaphragm by the quick-freezing and deep-etching replica method. Eur. J. Cell Biol. 53:402-406.

7. Kestilä, M., et al. 1998. Positionally cloned gene for a novel glomerular protein-nephrin-is mutat- ed in congenital nephrotic syndrome. Mol. Cell. 1:575-582.

8. Holthöfer, H., et al. 1999. Nephrin localizes at the podocyte filtration slit area and is characteristically spliced in the human kidney. Am. J. Pathol. 155:1681-1687.

9. Holzman, L.B., et al. 1999. Nephrin localizes to the slit pore of the glomerular epithelial cell. Kidney Int. 56:1481-1491.

10. Ruotsalainen, V., et al. 1999. Nephrin is specifically located at the slit diaphragm of glomerular podocytes. Proc. Natl. Acad. Sci. U. S. A. 96:7962-7967.

11. Lenkkeri, U., et al. 1999. Structure of the gene for congenital nephrotic syndrome of the Finnish type (NPHS1) and characterization of mutations. Am.J. Hum. Gen. 64:51-61.

12. Patrakka, J., et al. 2000. Congenital nephrotic syndrome (NPHS1): features resulting from different mutations in Finnish patients. Kidney Int. 58:972-980.

13. Putaala, H., Soininen, R., Kilpeläinen, P., Wartiovaara, J., and Tryggvason, K. 2001. The murine nephrin gene is specifically expressed in kidney, brain and pancreas: inactivation of the gene leads to massive proteinuria and neonatal death. Hum. Mol. Gen. 10:1-8.

14. Hamano, Y., et al. 2002. Determinants of vascular permeability in the kidney glomerulus. J. Biol. Chem. 277:31154-31162.

15. Reiser, J., Kriz, W., Kretzler, M., and Mundel, P. 2000. The glomerular slit diaphragm is a modified adherens junction. J. Am. Soc. Nephrol. 11:1-8.

16. Donoviel, D.B., et al. 2001. Proteinuria and perinatal lethality in mice lacking NEPH1, a novel protein with homology to NEPHRIN. Mol. Cell. Biol. 21:4829-4836.

17. Liu, G., et al. 2003. Neph1 and nephrin interaction in the slit diaphragm is an important determinant of glomerular permeabiliy. J. Clin. Invest. 112:209-221. doi:10.1172/JCI200318242.

18. Inoue, T., et al. 2001. FAT is a component of glomerular slit diaphragms. Kidney Int. 59:1003-1012.

19. Verma, R., et al. 2003. Fyn binds to and phosphorylates the kidney slit diaphragm component nephrin. J. Biol. Chem. 278:20716-20723.

20. Huber, T.B., Köttgen, M., Schilling, B., Walz, G., 
and Benzing, T. 2001. Interaction with podocin facilitates nephrin signaling. J. Biol. Chem. 276:41543-41546.

21. Shih, N.-Y., et al. 1999. Congenital nephrotic syndrome in mice lacking CD2-associated protein. Science. 286:312-315.

22. Schwarz, K., et al. 2001. Podocin, a raft-associated component of the glomerular slit diaphragm, interacts with CD2AP and nephrin. J. Clin. Invest. 108:1621-1629. doi:10.1172/JCI200112849.

23. Roselli, S., et al. 2002. Podocin localizes in the kidney to the slit diaphragm area. Am. J. Pathol. 160:131-139.

24. Saleem, M.A., et al. 2002. Co-localization of nephrin, podocin and the actin cytoskeleton. Evidence for a role in podocyte foot process formation. Am. J. Pathol. 161:1459-1466.

25. Barletta, G.-M., Kovari, I.A., Verma, R.K., Kerjaschki, D., and Holzman, L.B. 2003. Nephrin and Neph1 co-localize at the podocyte foot process intercellular junction and form cis hetero-oligomers. J. Biol. Chem. 278:19266-19271.

26. Gerke, P., Huber, T.B., Sellin, L., Benzing, T., and Walz, G. 2003. Homodimerization and heterodimerization of the glomerular podocyte proteins nephrin and NEPH1. J. Am. Soc. Nephrol. 14:918-926.

27. Khoshnoodi, J., et al. 2003. Nephrin promotes cellcell adhesion through homophilic interaction. Am. J. Pathol. 163:2337-2346.

28. McEwen, B.F., and Marko, M. 2001. The emergence of electron tomography as an important tool for investigating cellular ultrastructure. J. Histochem. Cytochem. 49:553-563.

29. Baumeister, W. 2002. Electron tomography: towards visualizing the molecular organization of the cytoplasm. Curr. Opin. Struct. Biol. 12:679-684.

30. Murk, J.L.A.N., et al. 2003. Endosomal compartmentalization in three dimensions: implications for membrane fusion. Proc. Natl. Acad. Sci. U. S. A 100:1332-1337.

31. Böhm, J., et al. 2000. Toward detecting and identifying macromolecules in a cellular context: Template matching applied to electron tomograms. Proc. Natl. Acad. Sci. U. S. A. 97:14245-14250.

32. Moritz, M., Braunfeld, M.B., Guénebaut, V., Heurser, J., and Agard, D.A. 2000. Structure of the Atubulin ring complex: a template for microtubule nucleation. Nat. Cell Biol. 2:365-370.

33. Medalia, O., et al. 2002. Macromolecular architecture in eukaryotic cells visualized by cryoelectron tomography. Science. 298:1209-1213.

34. Wetterberg, I., Zhao, J., Masich, S., Wieslander, L., and Skoglund, U. 2001. In situ transcription and splicing in the Balbiani ring 3 gene. EMBO J. 20:2564-2574.

35. Ziese, U., Kubel, C., Verkleij, A., and Koster, A.J. 2002. Three-dimensional localization of ultrasmall immuno-gold labels by HAADF-STEM tomography. J. Struct. Biol. 138:58-62.

36. Farquhar, M.G., Wissig, S.L., and Palade, G.E. 1961. Glomerular permeability. I. Ferritin transfer across the normal glomerular capillary wall. J. Exp. Med. 113:47-66.

37. Harris, L.J., Larson, S.B., Hasel, K.W., and McPherson, A. 1997. Refined structure of an intact IgG2a monoclonal antibody. Biochemistry. 36:1581-1597.

38. Bongini, L., et al. 2004. Freezing immunoglobins to see them move. Proc. Natl. Acad. Sci. U. S. A. 101:6466-6471.

39. Skoglund, U., Öfverstedt, L.-G., Burnett, R.M., and Bricogne, G. 1996. Maximum-entropy threedimensional reconstruction with deconvolution of the contrast transfer function: a test application with adenovirus. J. Struct. Biol. 117:173-188.

40. Studer, D., Michel, M., Wohlwend, M., Hunziker, E.B., and Buschmann, M.D. 1995. Vitrification of articular cartilage by high-pressure freezing. J. Microsc. 179:321-332.

41. Sugio, S., Kashima, A., Mochizuki, S., Noda, M., and Kobayashi, K. 1999. Crystal structure of human serum albumin at 2.5 Å resolution. Protein Eng. 12:439-446.

42. Carter, D.C., et al. 1989. Three-dimensional structure of human serum albumin. Science. 244:1195-1198.

43. Smithies, O. 2003. Why the kidney glomerulus does not clog: a gel permeation/diffusion hypothesis of renal function. Proc. Natl. Acad. Sci. U. S. A. 100:4108-4113.

44. Ruotsalainen, V., et al. 2000. Role of nephrin in cell junction formation in human nephrogenesis. Am. J. Pathol. 157:1905-1916.

45. Reeves, W., Caufield, J.P., and Farquhar, M.G. 1978 Differentiation of epithelial foot processes and filtration slits. Lab. Invest. 39:90-100.

46. Ryan, G.B., Rodewald, R., and Karnovsky, M.J. 1975. An ultrastructural study of the glomerular slit diaphragm in aminonucleoside nephrosis. $L a b$. Invest. 33:461-468.

47. Schneeberger, E.E., and Grupe, W.E. 1976. The ultrastructure of the glomerular slit diaphragm in autologous immune complex nephritis. Lab. Invest. 34:298-305.

48. Kurihara, H., Anderson, J.M., Kerjaschki, D., and Farquhar, M.G. 1992. The altered glomerular filtration slits seen in puromycin aminonucleoside nephrosis and protamine sulfate-treated rats contain the tight junctional protein ZO-1. Am. J. Pathol. 141:805-815.

49. Karnovsky, M.J., and Ryan, G.B. 1975. Substructure of the glomerular slit diaphragm in freeze-fractured normal rat kidney. J. Cell Biol. 65:233-236.

50. Schneeberger, E.E., Levey, R.H., McCluskey, R.T., and Karnovsky, M.J. 1975. The isoporous substructure of the human glomerular slit diaphragm. Kidney Int. 8:48-52.

51. Furukawa, T., et al. 1991. Morphometric study of glomerular slit diaphragms fixed by rapid-freezing and freeze-substitution. Kidney Int. 40:621-624.

52. Radice, G.L., et al. 1997. Precocious mammary gland development in P-cadherin-deficient mice. J. Cell Biol. 139:1025-1032.

53. Mitchell, K.J., et al. 2001. Functional analysis of secreted and transmembrane proteins critical to mouse development. Nat. Genet. 28:241-249.

54. Angst, B.D., Marcozzi, C., and Magee, A.I. 2001. The cadherin superfamily. J. Cell. Sci. 114:625-626.

55. Weis, W.I. 1995. Cadherin structure: a revealing zipper. Structure. 3:425-427.

56. Sellin, L., et al. 2003. NEPH1 defines a novel family of podocin interacting proteins. FASEB J. 17:115-117.

57. Boute, N., et al. 2000. NPHS2, encoding the glomerular protein podocin, is mutated in autosomal recessive steroid-resistant nephrotic syndrome. Nat. Genet. 24:349-354.

58. Schnabel, E., Anderson, J.M., and Farquhar, M.G. 1990. The tight junction protein ZO-1 is concentrated along slit diaphragms of the glomerular epithelium. J. Cell Biol. 111:1255-1263.

59. Kawachi, H., et al. 1995. Developmental expression of the nephritogenic antigen of monoclonal antibody 5-1-6. Am. J. Pathol. 147:823-833.

60. Huber, T.B., et al. 2003. The carboxyl terminus of neph family members binds to the PDZ domain protein zonula occludens-1. J. Biol. Chem. 278:13417-13421.

61. Simons, M., et al. 2001. Involvement of lipid rafts in nephrin phosphorylation and organization of the glomerular slit diaphragm. Am. J. Pathol. 159:1069-1077.

62. Kurihara, H., Anderson, J.M., and Farquhar, M.G. 1995. Increased Tyr phosphorylation of ZO-1 during modification of tight junctions between glomerular foot processes. Am. J. Physiol. 268:F514-F524.

63. Griffiths, G., McDowall, A., Back, R., and Dubochet, J. 1984. On the preparation of cryosections for immunocytochemistry. J. Ultrastruct. Res. 89:65-78.

64. Okret, S., Wikström, A.-C., Wrange, Ö., Andersson, B., and Gustafsson, J.-Å. 1984. Monoclonal antibodies against the rat liver glucocorticoid receptor. Proc. Natl. Acad. Sci. U. S. A. 81:1609-1613.

65. Miralles, F., et al. 2000. Electron tomography reveals posttranscriptional binding of pre-mRNPs to specific fibers in the nucleoplasm. J. Cell Biol. 148:271-282.

66. Sandin, S., Öfverstedt, L.-G., Wikström, A.-C., Wrange, Ö., and Skoglund, U. 2004. Structure and flexibility of individual immunoglobulin G molecules in solution. Structure. 12:409-415.

67. Skoglund, U., Öfverstedt, L.-G., and Daneholt, B. 1998. Procedures for three-dimensional reconstruction from thin sections with electron tomography. In RNP particles, splicing and autoimmune diseases. J. Schenkel, editor. Springer-Verlag. Berlin, Germany. 72-94.

68. Humphrey, W., Dalke, A., and Shulten, K. 1996. VMD: visual molecular dynamics. J. Mol. Graph. 14:33-38.

69. Liu, L., et al. 2001. Defective nephrin trafficking caused by missense mutations in the NPHS1 gene: insight into the mechanisms of congenital nephrotic syndrome. Hum. Mol. Genet. 10:2637-2644. 